\title{
Measurements of periodically electric field from the interior of the earth
}

\section{J. M. Tsatsaragos*}

Electronic Engineer, 67 Esfigmenitou Street, 38221, Volos, Hellas.

Received 22 April 2008; Accepted 17 November 2008

\begin{abstract}
This paper presents electric field measurements in the underground water horizon that have been processed by a specialised model installation which receives and records electric signals. From the description and the technical characteristics of the installation, it results that the origin of changes of the recorded electric field is owed to a natural process that concerns the interior of our planet. External factors such as the Ionosphere and human activity do not interfere with the measurements of this particular installation.

The presentation of the measurements is dominated by a permanent periodic variation of the electric field with a constant period of about 24 hours. The basic periodic variation of the electric component, which is recorded and examined here, is modulated by pulses of variable amplitude and duration, accidentally distributed in time. The possible generating mechanism of pulses is also under examination in this paper. The result of spectrum analysis of the initial recordings shows a high peak which is identified with the equivalent maximum of the two main mechanical tidal components that are described in the bibliography. It is under examination whether the measured changes are related or emanating from the tidal deformity of the ground.

Other available measurements of the components of the Earth's magnetic field (included in the measurements carried out by special scientific projects in Greece and around the world) show the existence of a permanent periodic variation with a constant period of about 24 hours. The time comparison between the variations of the vertical component of the Earth's magnetic field and the measured variations of the electric field reveals a phase shift of 90 degrees.

Taking into consideration the above statement, we may infer that the periodic variation of the measured electric signal is the electric component of an electromagnetic wave. It appears that this electromagnetic wave is caused by the tidal deformity of the Earth. This paper proposes a theoretical model that can justify the nature and the mechanism of genesis of that electromagnetic wave, including other electromagnetic waves emanating from the Earth's periodic tidal deformity
\end{abstract}

Keywords: Electric Field inside Underground Water Layer, ELF Natural Electromagnetic Emissions, Geo-Electromagnetic Radiations, Electromagnetic Radiation under Tidal Influence.

\section{Introduction}

Since 1981, the writer has had great experience in measuring, recording and analysing changes of the electric field received with the use of electrodes in the ground. During the years 1981-1998, the measurements were taken from a surface electric dipole and were continuous (on a 24hour basis).

In 1998, the writer began a continuous (24hour), digital measurement and recording of the electric field in the underground water horizon in a rural area in the region of Magnesia, Greece. The installation was completed in November 2000 with measurements in two directions in the underground water horizon.

From December 2000 till September 2002, the writer collaborated with Dr. C. Thanassoulas, geophysicist, working on earthquake prediction. The use of proportional and digital, band-pass filters in the initial recordings (still carried out until today in Magnesia) found out a component of variable amplitude with a period of 24 hours,

\footnotetext{
*E-mail address:tsats64@otenet.gr

ISSN: 1791-2377 ( 2008 Kavala Institute of Technology. All rights reserved.
}

Thanassoulas et al. [5], Thanassoulas [7], Thanassoulas and Tsatsaragos [27] (Fig.1).

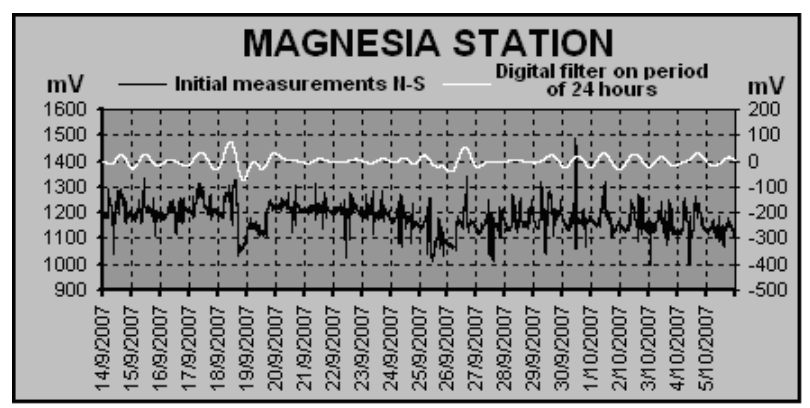

Fig. 1. Extract from the recordings of the Magnesia station.

Dr. Thanassoulas still believes that this 24 hour variation of the electric field is a seismic, precursory, electric signal, independent from the natural mechanism that generated it, Thanassoulas [7]. However, the big number of the spectrum components contained in the recordings of the station in Magnesia (Fig.2) does not drive to any positive or negative conclusion. 


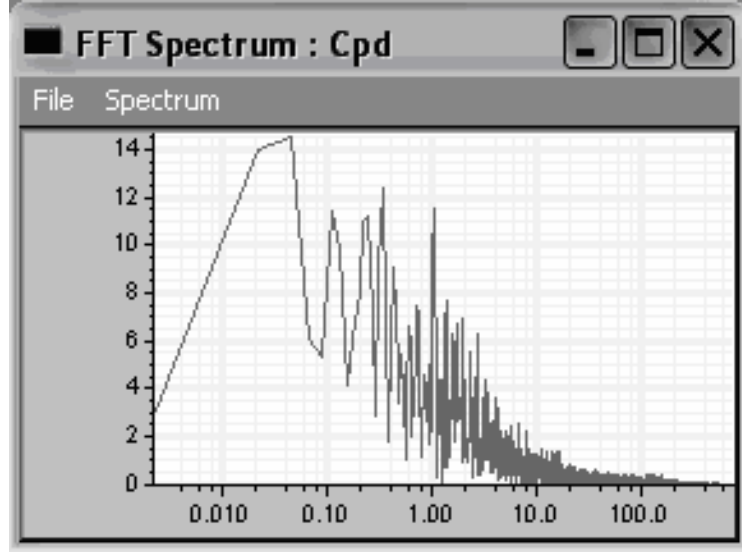

Fig. 2. Spectrum Analysis of the initial recordings in Figure 1 in circles per day.

In the beginning of 2007, the writer designed from scratch and then installed a new station of measurement in a rural area in the region of Messinia, Greece. The new installation was designed taking into consideration the evasion or rejection of any possible parasitic noise which could influence the measurements. Simultaneously, a similar model appliance of continuous recording was installed in the area of measurements in Magnesia, which remained in use for a small time interval.

In December 2007, the writer installed a model apparatus of measurement of the Earth's magnetic field in the area of Magnesia. The orientation of the installation is vertical to the axis of Earth.

The station in Messinia records a continuous daily periodic variation of the electric field of variable amplitude in time. The permanent nature of this daily periodic variation in a 24hour period recorded both in Magnesia and Messinia is the object of research presented onwards.

Other researchers also recording the electric field from the ground (but are focused mainly on periods of 100sec) argue that there are electromagnetic emissions from the ground in a wide area of the electromagnetic spectrum, Hata et al. [3], O'Keefe and Thiel [4], Gokhberg et al. [8], Kagiyama et al. [9], Hata et al. [10], [11], Fujinawa and Takahashi [12], Eftaxias et al. [14], Rokityanski [15], Hata and Yabashi [19], Kawada et al. [21], Eftaxias et al. [22], Mavrodiev [28].

\section{Arrangement of the station of measurement in Messinia}

\section{A. The external installation}

The station of measurement is installed in the countryside of Messinia, far away from other civil and industrial settlements. The installation uses the metal tubes of three drillings as the end of three electric dipoles. The three drilling holes are found on the tops of an almost right-angled triangle, the vertical sides of which is roughly 100 meters in length. Drilling 1 delimits the right angle of the triangle (Fig.3).

The layer of solid ground above the underground water layer is also electrically conductive and works as a Faraday shield against ionospheric or weather-influence signals. Therefore, it absorbs all parasitic interjection that may emanate from above and out of the underground water layer (such as leakages of electric grounding, man-made electromagnetic emissions, ionospheric emissions etc).

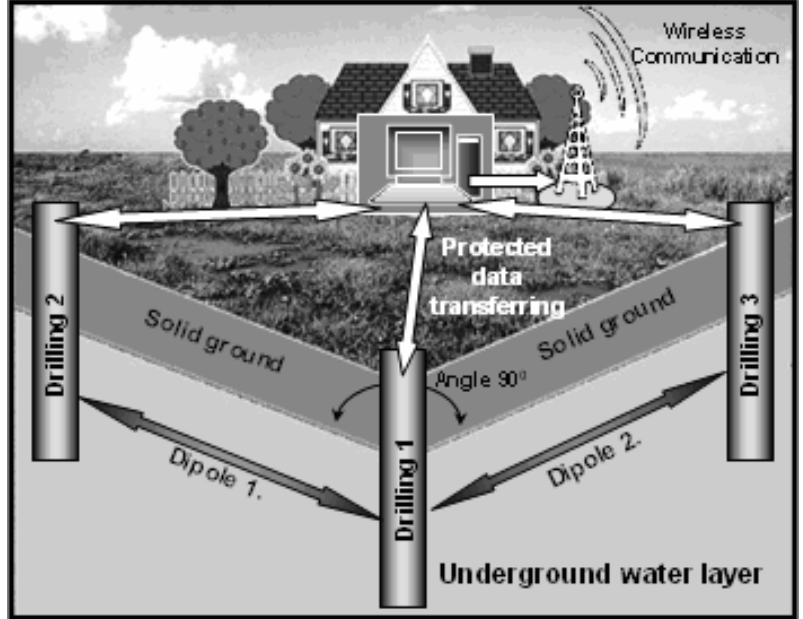

Fig 3. View of the installation of the Messinia Station.

The voltage difference between Dipoles 1 and 2 is the useful data which is recorded in the central installation within a small house. The data is recorded by a computer in daily digital files (24 hours a day).

Finally, the computer is connected to a special wireless communication network that allows both the remote control of the installation and the reception of the daily files from distance.

\section{B. Ground plan of the field of measurements}

The three drillings are found in a rural area of Messinia, far away from industrial activity, as was already reported. The drillings are simultaneously used for the needs of irrigation of adjacent cultivations. This ensures that they really lead into the water layer. The depth of each of the three drillings reaches 36 metres in the water layer. The two electric dipoles are oriented as it appears in Figure 4.

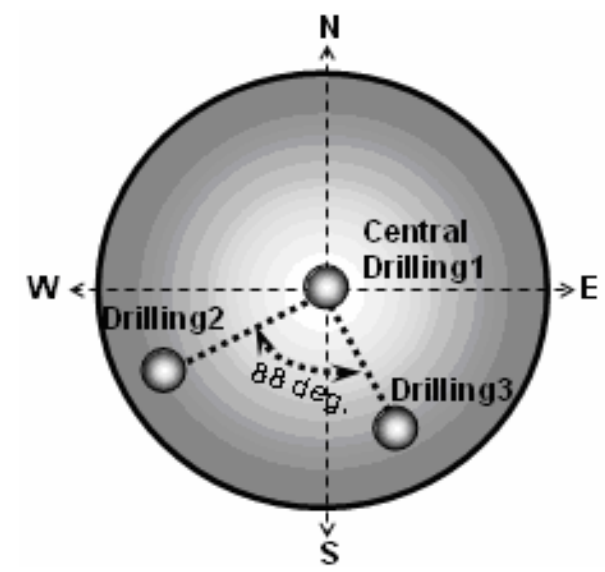

Fig. 4. Ground plan of the Messinia station drillings.

The precise length of each of the electric dipoles is as follows: 123 meters for the Dipole 1 and 87 meters for Dipole 2. The angle between them is 88 degrees. The conversion of measurements to values of equilateral dipoles, directed North-South and East-West, is converted mathematically at the time of data processing. 
C. The central installation - electronic structure. (Fig.5) The station includes:

a. The housing of the central installation (small house, Fig.5)

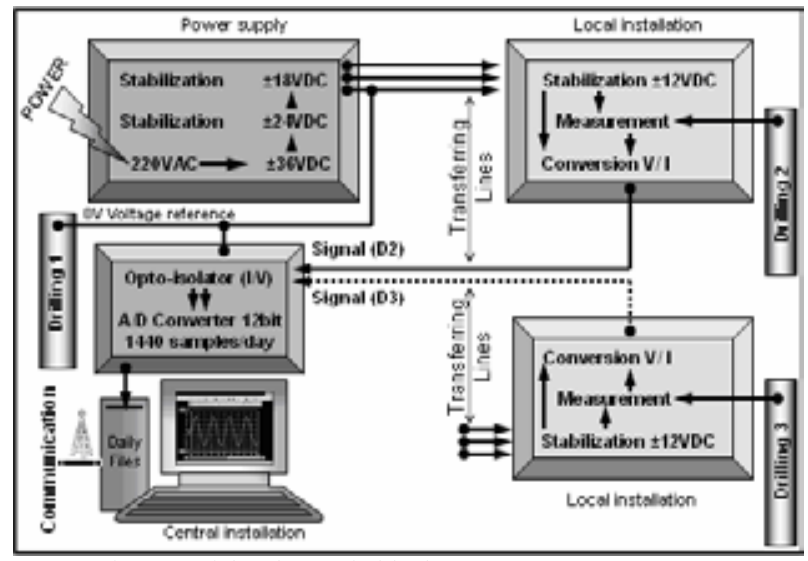

Fig. 5. Diagram of the electronic block.

It is placed very close to Drilling 1 which is found on the top of right-angled triangle and includes:

- Low voltage power supply of double successive reduction and stabilization

This ensures stable $\mathrm{D} / \mathrm{C}$ power supply used for the operation of all electronic stages, independent from changes of voltage of the A/C line. Simultaneously, all external, parasitic noise that could be transported in the system through the $\mathrm{A} / \mathrm{C}$ power line is totally rejected.

- Optical decouplers for each channel of measurements

These ensure the protection and the electric isolation between the computer and each stage of measurement.

\section{- A computer device (P/C)}

It includes an analog measurement card with $12 \mathrm{bit}$ Analog to Digital converter. The computer runs special software which was created by the writer exclusively for the storage of the measurements in daily files. Specialized programs running ensure the wireless, distant communication with the system, so that the complete remote control and reception of the files is achieved.

- A system of wireless communication

It is used for the complete remote control of the station.

\section{b. Local electronic block, electrically and magnetically} shielded (Fig.5)

This is found very close to each drilling and includes:

- Electronic voltage reducer - stabilizer for the local needs

This method excludes any external noise which could interfere with the low voltage power lines and ensures that noise is not transported to the circuits of measurement.

- The electronic circuit block of measurement

It measures the voltage difference of each drilling with respect to the central drilling (Drilling 1) located on the top of the right-angled triangle.

\section{- Voltage to current converter (V/I)}

The converter is indispensable for the integrity of the signal so that the signal transportation to the central installation is clear of noise. (Industrial method).

c. Underground multi-line cable of connection and transportation of data (big length)
This high endurance cable is placed underground for security reasons.

\section{The recordings from the station of measurement}

\section{A. The measurements}

Figure 6 is an extract from the measurements of the electric field concerning a five-day period recorded during September 2007. These are the initial measurements without any other kind of processing or the use of any electric or electronic filter.

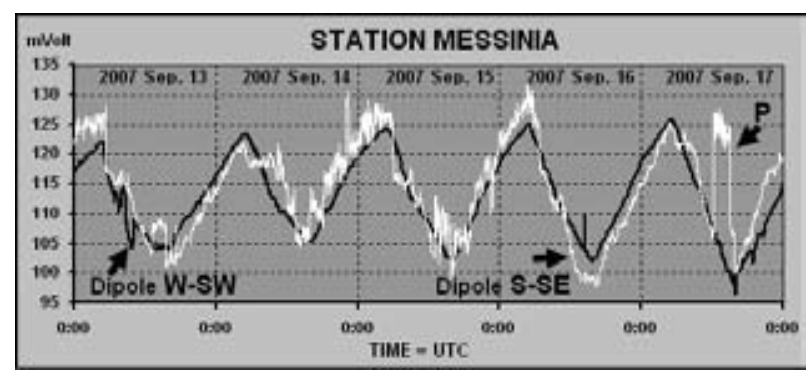

Fig. 6. Extract from the recordings of the Messinia station.

The black curve concerns Dipole 1 which is South-West oriented. The white curve concerns Dipole 2 which is SouthEast oriented. The horizontal axis depicts UTC time while the vertical axis presents the signal amplitude in mVolts.

What we observe in both dipoles is a basic periodic change with a period of about 24 hours. We also observe certain pulses of variable duration and amplitude that modulate the basic periodic curve, for example the one that is marked with the letter $(\mathrm{P})$ in the white curve.

It should be noted that the biggest value of the noise level measured during the tests was roughly $250 \mu$ Volts.

\section{B. Spectrum Analysis (in periods)}

What appears in Figures 7 and 8 is the result of the F.F.T. spectrum analysis concerning data for a time period of 122 days $(122 * 1440=175680$ values per dipole $)$. The data concerns the period between March 7 and April 16, 2007. The program used for the analysis was TSOFT of the Royal Observatory of Belgium. Magnifying the signal peak region we get Figure 8 .

Here we observe that the peak of the curve has a maximum of period of 23.93 hours in both dipoles, whereas it deviates at both sides for a few minutes (Fig.8).

However, the characteristic period of 23.93 hours is located in the components of the mechanic lithosphere oscillation (well-known to the geophysics) which is caused by the tidal gravity influence, Thanassoulas et al. [7], Boyce [13], Sazhina and Grushinsky [16], Garland [18], [24], (Tab.1).

Here we note that the maximum peak presented in Figures 7 and 8 belongs to the period of 23.93 hours and precisely coincides with the period of the Lunar-Solar declinational tidal component K1. The principal Solar declinational component $\mathrm{P} 1$ is also included in the signal peak region. 
Table 1

Primary tidal components

\begin{tabular}{c|lc}
\hline $\begin{array}{c}\text { Tidal } \\
\text { component }\end{array}$ & \multicolumn{1}{|c}{ Description } & $\begin{array}{c}\text { Period } \\
\text { (hours) }\end{array}$ \\
\hline J1 & Small Lunar Elliptic & 23.10 \\
\hline K1 & $\begin{array}{l}\text { Lunar-Solar } \\
\text { Declinational }\end{array}$ & 23.93 \\
\hline P1 & $\begin{array}{l}\text { Principal Solar } \\
\text { Declinational }\end{array}$ & 24.07 \\
\hline M1 & Smaller Lunar Elliptic & 24.86 \\
\hline O1 & $\begin{array}{l}\text { Principal Lunar } \\
\text { Declinational }\end{array}$ & 25.82 \\
\hline Q1 & Larger Lunar Elliptic & 26.87 \\
\hline
\end{tabular}

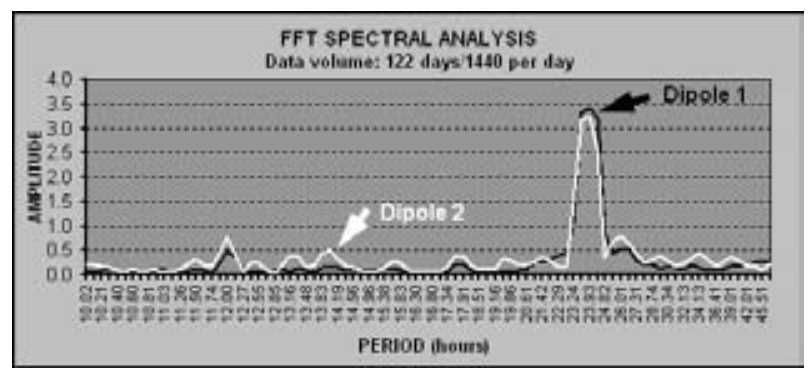

Fig. 7. F.F.T. spectrum analysis of the initial measurements.

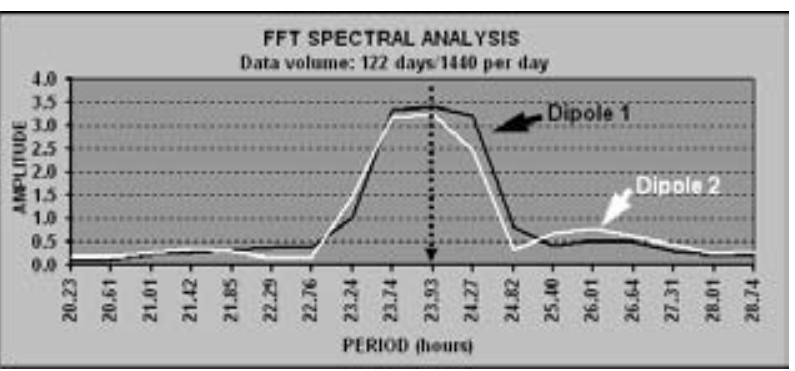

Fig. 8. Focus on the peak region of Fig.7.

The above statement places under further examination if there is any co-relation (and to what extent) between the recorded periodic changes of the electric field examined and the mechanic tidal oscillation of the Lithosphere.

\section{Can we measure the electric component of an electromagnetic wave?}

The question that arises then is: If we really have to do with an electromagnetic (E/M) wave, similar changes of the Earth's magnetic field must be measured with a phase difference of 90 degrees. However, the station in Messinia is not equipped with sensors suitable for measuring the Earth's magnetic field. As is widely known, every E/M wave is inductively received when the distance between emission and reception is smaller than half wavelength. In the case of an E/M wave with a period of about 24 hours, the wave length is roughly 670.000 times the length of the equator of the Earth. Consequently, when an E/M wave emanates from any point of the Earth, the measurements of its components should be recorded anywhere on the Earth's surface, because the distance between transmitter and receiver is, in all cases, much smaller than half wavelength. Thus, in the process of research, the writer consulted researchers measuring the Earth's magnetic field.
Among others, the Greek Earthquake Planning and Protection Organisation (O.A.S.P.) runs the following special project: "Multi-parametrical Observation of the Volcano in Nissiros Island". One of its tasks is the measurement of the Earth's magnetic field components. One measurement stations is located in Anavissos, an area close to Athens. An extract from the measurements of the Anavissos station was nobly given by Dr Dimitrios Foudoulis, tectonic geologist. These measurements of the components of the Earth's magnetic field also show a permanent periodic change with a constant period of about 24 hours (Fig.9). Unfortunately, the accuracy factor of these measurements is not satisfactory for the present research. Thus, these recordings can be taken into consideration only as indicative of the periodic change of the vertical component of the Earth's magnetic field.

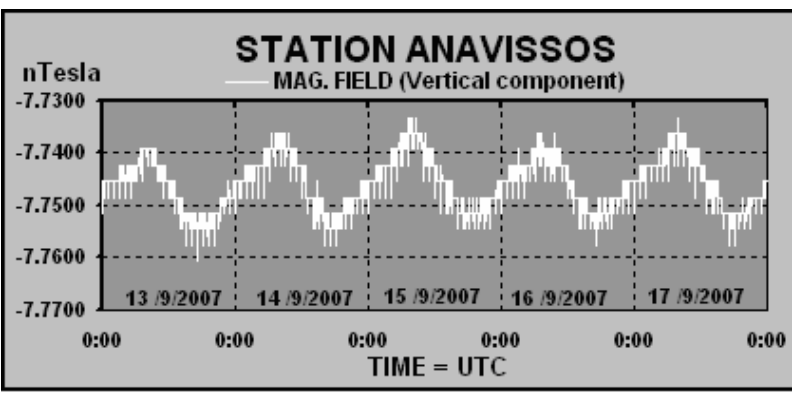

Fig. 9. Variations of the Earth's magnetic field.

What we observe in the Anavissos measurements are permanent periodic changes with a period of about 24 hours, similar to the periodic changes of the electric field recorded in Messinia. This is the first indication that both stations in Anavissos and Messinia describe the same phenomenon or phenomena emanating from the same source. This led to the manufacturing and installation of special appliance for measuring the Earth's magnetic field in the area of the Magnesia station.

During February 2007, both the station in Messinia and that in Magnesia (equipped with a similar model appliance of measurement of the electric field) were in simultaneous operation. A comparative graph of the recordings in Messinia and Magnesia is presented in Figure 10. The recordings are in phase and look almost alike.

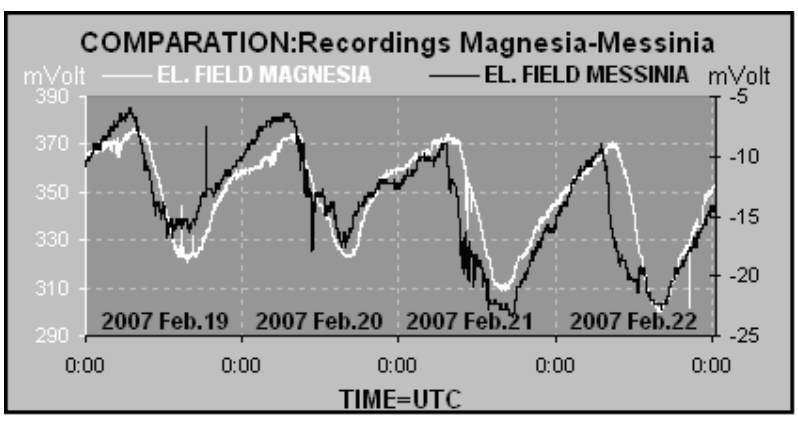

Fig. 10. Measurements from the Magnesia and Messinia stations taken using the same measuring apparatus.

Despite the fact that the philosophy behind the station of measurement of the electric field in Magnesia remains different and records some levels of noise (Fig.1), by applying the digital $1 \mathrm{c} / \mathrm{d}$ band pass filter, there is satisfactory depiction of the changes in a 24 hour time period.

In the measurements of the electric field in Magnesia and Messinia and in the corresponding measurements of the magnetic field in the Magnesia station, the same digital (1c/d) band pass filter was applied. The application of the 
filter resulted to comparable findings regarding the differences in phase. The result is the graph in Figure 11. In this graph, the values of the magnetic field are the original, while the values of the electric field (in Magnesia and Messinia) have been multiplied with suitable factors so that the three curves result to roughly the same amplitude.

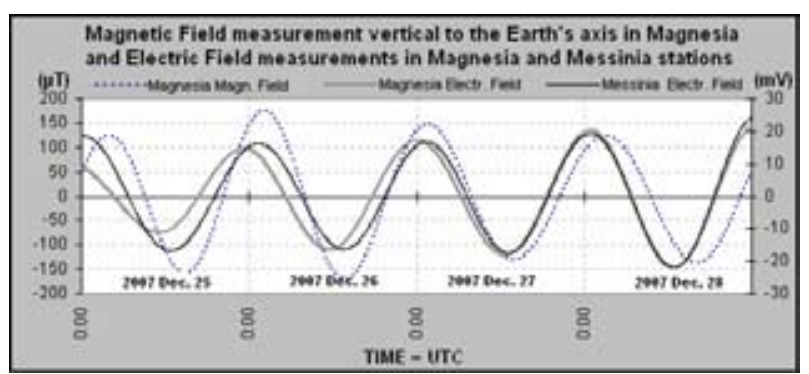

Fig 11. Phase comparison between the electric field and the magnetic field in Magnesia and the electric field in Messinia.

What we observe here is that all curves are almost in phase. The justification of the phenomenon is attempted in the theoretical approach presented onwards.

\section{Theoretical proposal Electromagnetic Wave Production (of a 24 hour period)}

The electric field between the surface of the Earth and the Ionosphere is well-known. The same field theoretically extends the interior of our planet to the boundary surface of the external metallic liquid core. Under the effect of the tidal Lunar-Solar gravitational forces on the Earth, its shape:

- $\quad$ is lengthened on the axis of these tidal gravitational forces $(\mathrm{AB})$. The longest part appears at the side of the effect. (OA > OB, Fig. 12)

- $\quad$ is shortened both equilaterally and vertically on the axis of the gravitational forces so that $(\mathrm{OC}=\mathrm{OD})$.

The oval shape of the Earth (ACBDA) is a deformity effect of the gravitational tidal forces. Due to this deformity:

- the intensity of the internal electric field on the side of the Lunar-Solar gravitational effect (OA) is smaller than that of the opposite side (OB). As a result, the electric field along the diameter $(\mathrm{AB})$ reaches its highest value (Arrow $\mathrm{AB}$ ).

- vertically to the middle point of the axis $(\mathrm{AB})$, the intensity of the internal electric field is increased equally at both sides of the axis. As a result, the total electric field between $\mathrm{C}$ and $\mathrm{D}$ is zero since the electric field between $\mathrm{O}$ and $\mathrm{C}$ is equal to the electric field between $\mathrm{O}$ and $\mathrm{D}$.

- finally, the total electric field exists along the arrow AB (Fig. 12).

Note: This is the typical phenomenon of a charged capacitor whose plates change in distance.

Under the effect of the total electric field, the electric charges (electrons) on the Earth's surface are orientated to the area around point B (the shape of half moon at B).

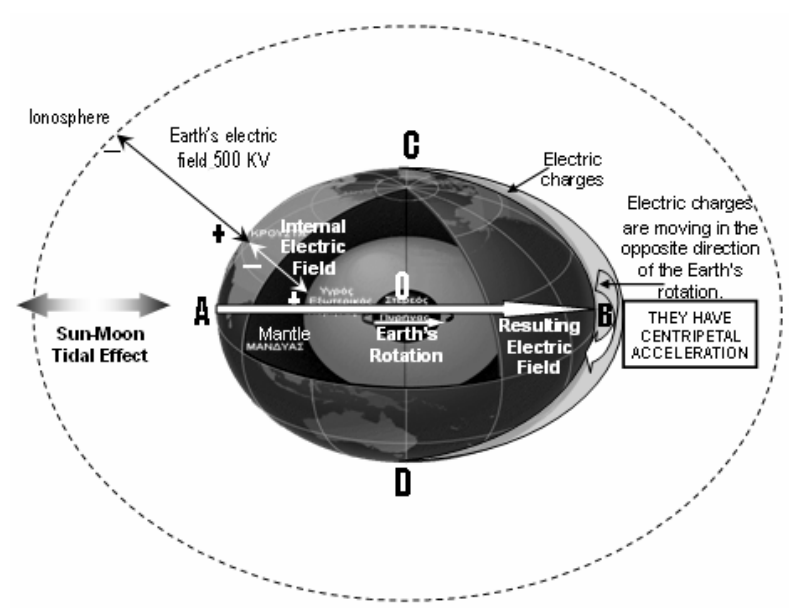

Fig. 12. Production mechanism of the elliptic E/M wave with a 24hour period.

Due to the rotation of the Earth, the electric charges at point $\mathrm{B}$ also rotate with the same velocity but in the opposite direction, in order to be always oriented in the electric field's direction. Because of the charges' circular movement, with a period of about 24 hours, they also have centripetal acceleration.

Under these circumstances (the motion of these electric charges with centripetal acceleration), an elliptical righthand, circularly polarized electromagnetic wave is generated, with a period equal to the period of the rotation of the Earth, according to Maxwell's theory (Fig.13). This electromagnetic wave is transmitted in space and is propagated in the direction of the Earth's rotation axis. Finally, we have a continuous power of the electromagnetic emission at point $\mathrm{B}$ which depends on the tidal deformity of the Earth.

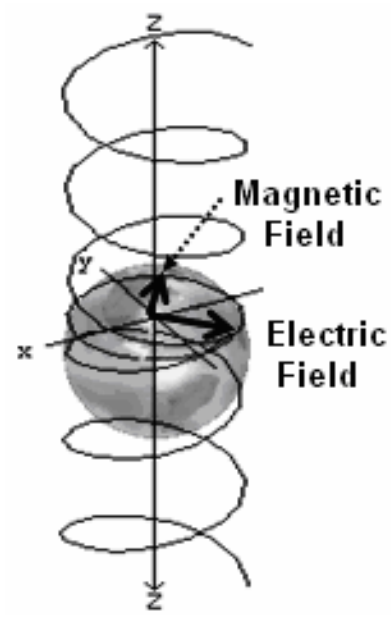

Fig. 13. Elliptically polarized E/M wave.

It appears that the electric component of this electromagnetic wave has a permanent periodic variation which dominates in the recordings of the Messinia station (Fig.6). The main characteristic of the elliptically polarized electromagnetic wave (Fig.13) is that the magnetic vector follows the electric vector with a phase difference of 90 degrees, Hata et al. [10], [11].

For the experimental examination of the difference in phase, the measurement of the Earth's magnetic field must be vertical to the Earth's rotation axis. This was taken into consideration when the installation of the Earth's magnetic 
field measurement apparatus took place at the area of the Magnesia station during the first days of December 2007.

Figure 14 presents a cut view of the Earth on a parallel circle level as it would be seen from the North Pole. Points 1 and 2 are on the horizontal plane of the place where the electric field component is measured. The figure also shows the vectors of the components of both the electric and the magnetic field.

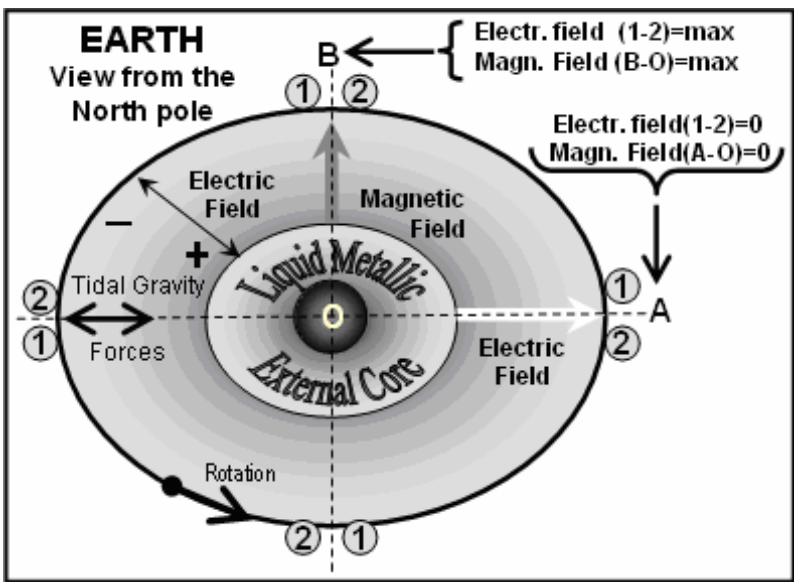

Fig. 14. Expected phase difference between the electric and the magnetic filed.

When the measurements are taken at point $\mathrm{A}$ (in the opposite side of the tidal gravitational forces), both the measured electric and magnetic components of the elliptically polarized electromagnetic wave must be zero, because their direction is vertical to the corresponding vectors. On the contrary, 6 hours later, when the place of measurements is at point $\mathrm{B}$, both measured electric and magnetic components of the elliptically polarized electromagnetic wave must be maximum, because their direction is parallel to the corresponding vectors. Thus, we must expect that the recorded components of both the electric and the magnetic field are in phase. Indeed, the measurements taken confirm this (Fig. 11).

Figure 15 presents a section of the Earth's interior. We can view the Asthenoshere, the solid upper mandle and the outside solid crust. We can also view the internal electric field which is an extension or part of the atmospheric electric field.

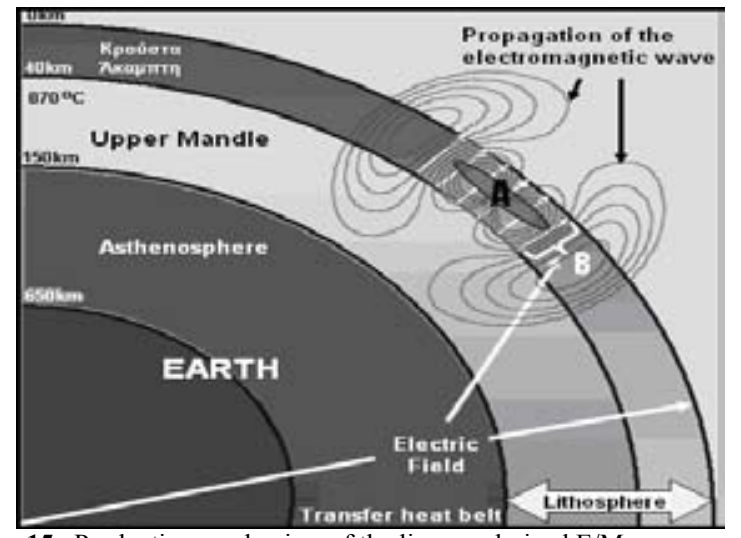

Fig. 15. Production mechanism of the linear polarized E/M wave.

Let us suppose that there are geological changes in the elliptic part of the crust (A), such as sudden movements of materials, cracks, humidity changes, alternations in pressure and temperature, etc. Any such change would cause some variation in the dielectric constant of the particular area. This would also cause a change in the electric field (B) and would produce an electromagnetic wave, according to Maxwell's theory.

The electromagnetic wave:

- would seem to be produced by a vertical transmitting antenna and is linear polarized.

- $\quad$ is propagated right-angled to the electric field lines and is parallel to the Earth's radius.

- lasts while the change in the electric field continues.

Figure 16 presents the electric and the magnetic field components of one linear polarized electromagnetic wave. Axis yy' represents the electric field while axis $z z$ ' the magnetic; axis $\mathrm{xx}^{\prime}$ is the axis of propagation of the electromagnetic wave. The direction of the source of this particular type of electromagnetic wave (Fig.15 and Fig.16) is calculated with the use of the radio directional finding method.

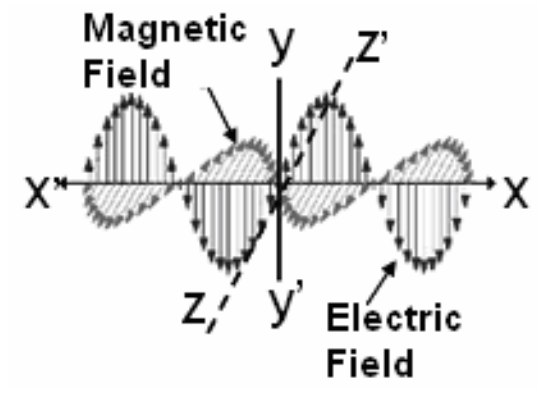

Fig. 16. Linear polarized $\mathrm{E} / \mathrm{M}$ wave.

The pulses shown in the original recordings of the electric field in the station of Messinia refer to one such linear polarized electromagnetic wave. Such a pulse is represented with the letter $P$.

\section{Summary - Conclusion}

The writer has designed, manufactured and installed a model measurement system of the electric field existing in the underground water horizon.

- The layer of solid ground above the underground water layer works as a Faraday shield dramatically reducing the ionospheric or other spurious, parasitic emissions emanating from above and out of the underground water.

- Changes of voltage in the $\mathrm{A} / \mathrm{C}$ power line and other spurious, parasitic emissions do not interfere with the system's measurement apparatuses.

- There is no parasitic interference on the cables of connection and transportation.

- The system records and stores data 24 hours a day, all year long.

- The installation is fully remotely controlled.

The model installation records a basic periodic variation of the electric field in the underground water horizon. By means of Fourier analysis, the original recordings present a maximum peak between the periods from 23.93 to 24.07 hours, both of which can be identified with the two components of the tidal deformity of the Earth. Shorter pulses of the basic period appear to modulate the basic periodic change.

The Magnesia station records a periodic change of the electric field in the underground water horizon similar to that of Messsinia. But, because of the different design in this 
station of measurement, the periodic change of the electric field is achieved by means of a digital F.F.T. filter.

During research, measurement apparatuses of the Earth's magnetic field (right-angled to the Earth's rotational axis) were installed and the recordings were added to the original measurements in the Magnesia station.

From a theoretical point of view:

- It seems that the measured periodic change of the electric field is possibly the electric component of a circular or an elliptically polarized electromagnetic wave emanating from the tidal deformity of our planet. The reception of the electromagnetic wave in a 24 hour period is inductive. This means that there is no attenuation between the source and the reception point.

- Natural processes in the interior of Earth definitely cause changes in the dielectric constant of the particular areas, producing the emission of a linear polarized electromagnetic wave. This wave seems to be transmitted due to changes of the continuous vertical electric field in the interior of Earth calculated by means of use of the radio directional finding method.

- The short duration pulses that modulate the basic periodic change seem to come from the linear polarized electromagnetic emission.

\section{The Future}

The research and its results belong to the writer himself without any other scientific, material or economic help. Fortunately, it continues to upgrade using all available means. Therefore, official scientific support of the research would be welcome, as it would offer great help in scientific explanation and the validation of the results, since the continuation of the research is highly demanding at all levels.

The writer is about to complete the assembly of the appropriate hardware in order to assure that the measurements in both Magnesia and Messinia stations are done in the same way. Having in mind what was observed in the Anavissos station, the writer manufactured and brought into operation a measurement apparatus of the Earth's magnetic field in the Magnesia station in December 2007. (The apparatus uses the KMZ10A Phillips sensor).

The infrastructure behind the operation of the two stations records and evaluates other parameters that could be useful at some point. The complete measuring installation of the Earth's magnetic field is already under construction in both stations.

Measurements of gravity acceleration can also be useful since daily gravity variations are caused by the tidal gravitational forces that critically influence tidal distortion.

Applying all the necessary programmed changes, the writer hopes to complete and confirm the theoretical model concerning electromagnetic emissions and will have in hand possible concussions for future applications that could be used in:

- The effect of tides on the internal structure of the Earth.

- Earth's interior activity related to volcanic activity.

- Earth's interior activity related to earthquakes.

The collection of new data and the latest observations in the process of research will be the subject of a future presentation in the forthcoming Greek Physics Association conference.
1. http://yako.physics.upatras.gr/waves/11.pdf

2. http://www.des.upatras.gr/physics/kounavis/sem2/electrmagn1.ht $\mathrm{m}$

3. M. Hata, I. Takumi, S. Yabashi and X. Tian, An Anomaly of ELF Band Vertical Magnetic Flux as a Precursor of Dome Formation at Unzen Volcano and its Model Analysis, Physics of the Earth and Planetary Interiors 105, 271-277 (1998).

4. S. G. O'Keefe and D. V. Thiel, A Mechanism for the Production of Electromagnetic Radiation during Fracture of Brittle Materials, Physics of the Earth and Planetary Interiors 89, 127-135 (1995).

5. C. Thanassoulas J. Tsatsaragos and V. Klentos, Determination of the Most Probable Time of Occurrence of a Large Earthquake, Open File Report A. 4338, IGME, Athens (2001).

6. J. Muto, and H. Nagahama, Dielectric Anisotropy and Deformation of Crustal rocks: Physical Interaction Theory and Dielectric Mylonites, Physics of the Earth and Planetary Interiors 141, 27-35 (2004).

7. C. Thanassoulas, Earthquake Prediction based on Electrical Signals recorded on Ground Surface. www.earthquakeprediction.gr/

8. M. B. Gokhberg, V. A. Mogounov and A. O. Pokhotelov, Earthquake Prediction: Seismo-Electromagnetic Phenomena, Gordon and Breach Publishers, Singapore (1995).

9. T. Kagiyama, H. Utada and T. Yamamoto, Electromagnetic Structure and Magma Ascending Process, in Unzen Volcano: The 1990-1992 Eruption, (Eds.) K. Ohta, Nishinippon and Kyushu University Press, Fukuoka p.74-79 (1995).

\section{References}

10. M. Hata, I. Takumi, S. Yabashi, and X. Tian, Electromagneticwave Radiation due to Diastrophism of Magma Dike Growth in Izu-Miyake Volcanic Eruptions in Japan in 2000, Aichi Prefectural University, Nagakute, Japan, 480-1198. www.nathazards-earth-syst-sci.net/1/43/2001/nhess-1-43-2001.pdf

11. www.nature.com/nature/journal/v347/n6291/abs/347376a0.html

12. Y. Fujinawa and K. Takahashi, Emission of Electromagnetic Radiation Preceding the Ito Seismic Swarm of 1989, Nature 347, 376-378 (1990).

13. E. Boyce, Observations, Empirical Studies and Harmonic Analysis, Web project for PHYS645 Fundamentals of Geophysical Fluid Dynamics, University of Alaska Fairbanks (2003).

http://ffden2.phys.uaf.edu/645fall2003_web.dir/Ellie_Boyce/harm onic.htm

14. K. Eftaxias, V. E. Panin and Ye. Ye. Deryugin, Evolution - EM Signals before Earthquakes in Terms of Mesomechanics and Complexity, Tectonophysics 431, 273-300 (2007).

15. I. I. Rokityanski, Geoelectromagnetic Investigation of the Earth's Crust and Mantle, Springer-Verlag (1982).

16. N. Sazhina and N. Grushinsky, Gravity Prospecting Change of Gravity with Time Tides, MIR Publishers, Moskow p.109-121 (1971).

17. S. Mares, Introduction to Applied Geophysics, D.Reidel Publishing Company, Dordrecht, Boston, Lancaster (1984).

18. G. Garland, Introduction to Geophysics: Mantle, Core and Crust, W. B Saunders Company, Washington p.198-206 (1971). 
19. M. Hata and S. Yabashi, Observation of ELF Radiation related to Volcanic and Earthquake Activities in Electromagnetic Phenomena related to Earthquake Prediction, (Eds.) M. Hayakawa and Y. Fujinawa, Terra Scientific (TERAPUB), Tokyo 159-174 (1994).

20. www.utdallas.edu/ cantrell/ee6334/polarization.pdf

21. Y. Kawada et al., Preseismic Electromagnetic Radiation and Magnetization, Department of Geoenvironmental Sciences, Graduate School of Science, Tohoku University, Sendai, Japan. www.nat-hazards-earth-syst-sci.net/7/599/2007/nhess-7-5992007.pdf

22. K. Eftaxias, P. Frangos, P. Kapiris, J. Polygiannakis, J. Kopanas and A. Peratzakis, Review and a Model of Pre-seismic Electromagnetic Emissions in terms of Fractal Electrodynamics, Fractals 12, 243-273 (2004).

23. J. M. Herndon, Sub-structure of the Inner Core of the Earth, Proceedings of the National Academy of Sciences 93, 646-648 (1996). www.pnas.org/cgi/reprint/93/2/646.pdf
24. www.phy.ornl.gov/csep/CSEP/OM/NODE31.html

25. V. Dehant, Tidal Parameters for an Inelastic Earth, Physics of the Earth and Planetary Interior 49, 97-116 (1987).

26. Y. Sasai, M. Ueshima, H. Utada, T. Kagiyama, J. Zlotnicki, T. Hashimoto and Y. Takahashi, The 2000 Activity of Miyakejima Volcano as Inferred Electric and Magnetic Field Observations, Journal of Geography 110, 2, 226-244 (2001).

27. C. Thanassoulas and J. Tsatsaragos, The Earthquakes of Izmit, Turkey $(\mathrm{Ms}=7.5 \mathrm{R}, 17-08-99)$ and Athens, Greece $(\mathrm{Ms}=5.9 \mathrm{R}$, 07-09-99) as were detected by Precursory Electrical Signals (24hr Oscillations) at Volos Area, Central Greece, IGME, Open File Report A. 3906, Athens (2000).

28. S. Cht. Mavrodiev, The Electromagnetic Fields under, on and up Earth Surface as Earthquakes Precursor in the Balkans and Black Sea Regions, (2002). http://arxiv.org/ 